\title{
in tinbergen
}

TI 2019-0072/II

Tinbergen Institute Discussion Paper

\section{Axiomatizations of the proportional division value}

Zhengxing Zou ${ }^{1}$

Rene van den Brink ${ }^{2}$

Youngsub Chun ${ }^{3}$

Yukihiko Funaki ${ }^{4}$

${ }^{1}$ Department of Econometrics and Operations Research, VU University, Amsterdam, The Netherlands, and School of Management and Economics, Beijing Institute of Technology, China

2 Department of Econometrics and Operations Research and Tinbergen Institute, VU University, Amsterdam, The Netherlands

3 Department of Economics, Seoul National University, Seoul, Korea

${ }^{4}$ School of Political Science and Economics, Waseda University, Tokyo, Japan 
Tinbergen Institute is the graduate school and research institute in economics of Erasmus University Rotterdam, the University of Amsterdam and VU University Amsterdam.

Contact: discussionpapers@tinbergen.nl

More TI discussion papers can be downloaded at https://www.tinbergen.nl

Tinbergen Institute has two locations:

Tinbergen Institute Amsterdam

Gustav Mahlerplein 117

1082 MS Amsterdam

The Netherlands

Tel.: +31(0)205984580

Tinbergen Institute Rotterdam

Burg. Oudlaan 50

3062 PA Rotterdam

The Netherlands

Tel.: +31(0)10408 8900 


\title{
Axiomatizations of the proportional division value
}

\author{
Zhengxing Zou ${ }^{\mathrm{a}, \mathrm{b}}$, René van den Brink ${ }^{\mathrm{a}}$, Youngsub Chun ${ }^{\mathrm{c}}$, Yukihiko Funaki ${ }^{\mathrm{d}}$ \\ ${ }^{a}$ Department of Econometrics and Operations Research, and Tinbergen Institute, VU \\ University, De Boelelaan 1105, 1081 HV Amsterdam, The Netherlands \\ ${ }^{b}$ School of Management and Economics, Beijing Institute of Technology, 100081 Beijing, \\ China \\ ${ }^{c}$ Department of Economics, Seoul National University, Seoul 08826, Korea \\ ${ }^{d}$ School of Political Science and Economics, Waseda University, 1-6-1 Nishi-Waseda, \\ Shinjuku-Ku, Tokyo 169-8050, Japan
}

\begin{abstract}
We present axiomatic characterizations of the proportional division value for TU-games, a value that distributes the worth of the grand coalition in proportion to the stand-alone worths of its members. First, a new proportionality principle, called balanced treatment, is introduced by strengthening Shapley's symmetry axiom, which states that if two players make the same contribution to any nonempty coalition, then they receive the amounts in proportion to their stand-alone worths. We characterize the family of values satisfying efficiency, weak linearty, and balanced treatment. We also show that this family is incompatible with the dummy player property. However, we show that the proportional division value is the unique value in this family that satisfies the dummifying player property. Second, we propose three appropriate monotonicity axioms by considering two games in which the stand-alone worths of all players are equal or in the same proportion to each other, and obtain three axiomatizations of the proportional division value without both weak linearity and the dummifying player property. Third, from the perspective of a variable player set, we show that the proportional
\end{abstract}

Email addresses: z.zou@vu.nl (Zhengxing Zou), j.r.vanden.brink@vu.nl (René van den Brink), ychun@snu.ac.kr (Youngsub Chun), funaki@waseda.jp (Yukihiko Funaki) 
division value is the only one that satisfies proportional standardness and projection consistency. Finally, we provide characterizations of proportional standardness.

Keywords: Cooperative game, proportional division value, monotonicity, consistency

JEL: C71

\section{Introduction}

A situation in which a finite set of players can generate certain worths by cooperation can be discribed by a cooperative game with transferable utility, or simply TU-game. A TU-game consists of a set of players and a characteristic function that specifies a worth to each coalition of players. A single-valued solution or value on a class of TU-games assigns a payoff vector to every game in this class.

The equal division value and the equal surplus division value are two wellknown equal surplus sharing solutions, which rely on egalitarian considerations. The equal division value allocates the worth of the 'grand coalition' (being the coalition consisting of all players) equally among all players. The equal surplus division value, also known as center-of-gravity of the imputation-set value in Driessen and Funaki (1991), gives each player its own stand-alone worth and an equal share of the remainder. Nevertheless, in many actual situations, and because of external and internal features of the players, proportionality sometimes seems a more appropriate equity principle. The idea of proportionality can be traced at least as far back as Aristotle's celebrated maxim, "Equals should be treated equally, unequals unequally, in proportion to relevant similarities and differences" from Nicomachean Ethics. With a natural proportionality consideration, the proportional rule 1 (Moriarity 1975 ; Banker 1981) allocates the worth of the grand coalition in proportion to the

\footnotetext{
${ }^{1}$ The proportional rule is identical to the stand-alone-coalition proportional value in Kamijo and Kongo (2015).
} 
stand-alone worths of its members. Thus, we call it the proportional division value, shortly denoted by $\mathrm{PD}$ value, which also in order to distinguish it from the proportional rule in claim problems, bargaining problems, insurance, law and so on. For other proportional solutions, we refer to the proportional value (Ortmann 2000; Khmelnitskaya and Driessen 2003; Kamijo and Kongo 2015), the proper Shapley values (Vorob'ev and Liapunov 1998; van den Brink et al. 2015), and the proportional Shapley value (Béal et al. 2018; Besner 2019) ? $^{2}$

The PD value depends only on the worths of one-person coalitions and the grand coalition, but ignores the worths of any other intermediate coalitions. Moulin (1987) characterizes the PD value for joint venture games, a class of TU-games where intermediate coalitions are inessential, i.e., their Harsanyi dividends are zero. These are the quasi-additive games in Carreras and Owen (2013), where the PD value is discussed by comparing with the Shapley value (Shapley 1953). Banker (1981) considers the situation that the worth of a coalition is a non-negative strictly increasing function with respect to the sum of the worths of its members. However, for more general TU-games, since the proportionality principle is not obvious, as far as we know, an axiomatic characterization of the PD value was still missing.

In this paper, we consider the problem of axiomatizing the $\mathrm{PD}$ value on the domain of TU-games in which the worths of all singleton coalitions have the same sign. This restrictive class of TU-games includes many applications, see Béal et al. (2018). We focus on some intuitive fairness criteria that widely used in the value theory for TU-games, including equal treatment of equals, monotonicity, and consistency. Our contributions can be discribed as follows.

We begin by introducing a proportionality principle called balanced treatment in TU-games, which is a strengthening of Shapley's symmetry axiom.

\footnotetext{
${ }^{2}$ We remark that the proportional division value cannot be considered as a weighted division value (Béal et al. 2016) or the weighted surplus division value (Calleja and Llerena (2017, 2019) ) since those values are based on exogenous weights, while the weights in the $\mathrm{PD}$ value are determined in the game, specifically they are equal to the stand-alone worths.
} 
This axiom states that the payoffs to two weak symmetric players, i.e., the contribution of these players to any nonempty coalition is the same, are proportional to the stand-alone worths of each other. It well captures the principles of 'equal treatment of equals' and 'unequal treatment of unequals'. Besner (2019) gives a similar axiom for the proportional Shapley value. Interestingly, balanced treatment together with efficiency and weak linearity, being a weak version of linearity introduced in Béal et al. (2018), give a family of values that have a formula similar as the family of efficient linear and symmetric values (ELS values for short) introduced in Ruiz et al. (1998), but the role of equal division replaced by proportional division. While the Shapley value is singled out from ELS values by employing the dummy player property, we reveal that there is no value belonging to our family that satisfies the dummy player property. Instead, we adopt the dummifying player property introduced in Casajus and Huettner (2014) and somewhat surprisingly obtain a characterization of the PD value.

We also provide three characterizations of the $\mathrm{PD}$ value by applying monotonicity axioms. A monotonicity axiom states that the payoff of a player should not decrease if a TU-game changes in certain ways that are 'advantageous' for this player. We introduce three such monotonicity axioms that are a relaxation of three existing axioms, by adding restrictions on the stand-alone worths of the players 3 The three existing axioms are coalitional monotonicity due to van den Brink (2007), which focuses on the worths of coalitions and is closely related to strong monotonicity in Young (1985), coalitional surplus equivalence and coalitional surplus monotonicity both axioms due to Casajus and Huettner (2014), which focus on the surplus of every coalition, i.e., the difference of the worth of a coalition and the total stand-alone worths of its members. Under efficiency and symmetry, van den Brink (2007) proved that coalitional monotonicity characterizes the equal

\footnotetext{
${ }^{3}$ This modification is similar in spirit to parameterized monotonicity introduced in Yokote and Funaki (2017).
} 
division value, and Casajus and Huettner (2014) proved that either coalitional surplus equivalence or coalitional surplus monotonicity characterizes the equal surplus division value. Not surprisingly, any of our monotonicity axioms together with efficiency and symmetry cannot characterize a unique value. Outstandingly, replacing symmetry by balanced treatment and keeping efficiency, we derive that any of our monotonicity axioms characterizes the PD value.

For a variable player set, we provide an axiomatization of the $\mathrm{PD}$ value using proportional standardness and the well-known projection consistency due to van den Brink and Funaki (2009). Proportional standardness requires to apply proportional division for two-player games, and is used in, e.g. Ortmann (2000), Khmelnitskaya and Driessen (2003) and van den Brink and Funaki (2009). Like other standardness axioms, proportional standardness is rather strong since it sets the payoff distribution for two-player games. Therefore, we conclude with characterizing the PD value on the class of twoplayer games. It turns out that grand worth additivity, the inessential game property, and continuity characterize proportional standardness.

The paper is organized as follows. Sect. 2 provides basic definitions and notation. In Sect. 3, we introduce balanced treatment and provide some results including an axiomatic charaterization of the PD value. In Sect. 4, we offer three axiomatic charaterizations using some appropriate monotonicity axioms. In Sect. 5, we give an axiomatic charaterization on variable player sets by employing projection consistency and proportional standardness. In Sect. 6, we characterize the PD value for two-player games. Finally, there is an appendix with the proof of Theorem 1 and the independence of the axioms in the characterization results.

\section{Preliminaries}

\subsection{Notation and TU-games}

We denote by $\mathbb{R}$ and $\mathbb{R}_{+}$the sets of all real numbers and positive real numbers, respectively. The cardinality of a set $S$ will be denoted by $|S|$ or, 
if no ambiguity is possible, appropriate small letter $s$. The notation $S \subseteq T$ means that $S$ is a subset of $T$, while the notation $S \subset T$ means that $S$ is a proper subset of $T$.

Let $\mathcal{U}$ be a fixed and infinite universe of players, and denote by $\mathcal{N}$ the set of all finite subsets of $\mathcal{U}$. A cooperative game with transferable utility, or simply a $T U$-game, is a pair $(N, v)$, where $N \in \mathcal{N}$ is the set of players, and $v: 2^{N} \rightarrow \mathbb{R}$ is the characteristic function assigning a worth $v(S)$ to each $S \in 2^{N}$, with the convention that $v(\emptyset)=0$. A subset $S \subseteq N$ or $S \in 2^{N}$ is called a coalition, and the associated real number $v(S)$ is the reward that coalition $S$ can guarantee by itself without the cooperation of the other players.

Denote $\mathcal{G}$ as the class of all TU-games with a finite player set in $\mathcal{N}$, and $\mathcal{G}^{N}$ the class of TU-games with player set $N$. Following Béal et al. (2018), a TU-game $(N, v)$ is individually positive if $v(\{i\})>0$ for all $i \in N$, and individually negative if $v(\{i\})<0$ for all $i \in N$. We express the notation $\mathcal{G}_{0}$ as the class containing all individually positive and individually negative TU-games, and $\mathcal{G}_{0}^{N}$ as the intersection of $\mathcal{G}_{0}$ and $\mathcal{G}^{N}$. For brevity, we refer to a TU-game just as a game.

\subsection{Values}

A value on $\mathcal{G}^{N}$ (respectively on $\mathcal{G}_{0}^{N}$ ) is a function $\psi$ that assigns a single payoff vector $\psi(N, v) \in \mathbb{R}^{N}$ to every game $(N, v) \in \mathcal{G}^{N}$ (respectively $(N, v) \in$ $\left.\mathcal{G}_{0}^{N}\right)$.

The equal division value is the value $\mathrm{ED}$ on $\mathcal{G}^{N}$ given by

$$
E D_{i}(N, v)=\frac{1}{n} v(N)
$$

for every $(N, v) \in \mathcal{G}^{N}$ and $i \in N$.

The proportional division value is the value $\mathrm{PD}$ on $\mathcal{G}_{0}^{N}$ given by

$$
P D_{i}(N, v)=\frac{v(\{i\})}{\sum_{j \in N} v(\{j\})} v(N)
$$

for every $(N, v) \in \mathcal{G}_{0}^{N}$ and $i \in N$. 
We employ the following definitions. Player $i \in N$ is a dummy player in game $(N, v)$ if $v(S \cup\{i\})=v(S)+v(\{i\})$ for all $S \subseteq N \backslash\{i\}$. Player $i \in N$ is a dummifying player in game $(N, v)$ if $v(S)=\sum_{j \in S} v(\{j\})$ for all $S \subseteq N$ with $i \in S$. Notice the difference between a dummy and dummifying player: a dummy player adds its own stand-alone worth when it joins any coalition, while a dummifying player entering a coalition results in the worth of that coalition becoming equal to the sum of the stand-alone worths of the players in that coalition. Players $i, j \in N, i \neq j$, are symmetric in $(N, v)$ if $v(S \cup\{i\})=v(S \cup\{j\})$ for all $S \subseteq N \backslash\{i, j\}$. For $(N, v),(N, w) \in \mathcal{G}_{0}^{N}$ and $a, b \in \mathbb{R}$, the game $(N, a v+b w)$ is defined by $(a v+b w)(S)=a v(S)+b w(S)$ for all $S \subseteq N$.

In the following, we state some properties of values for games.

- Efficiency. $\sum_{i \in N} \psi_{i}(N, v)=v(N)$ for all $(N, v) \in \mathcal{G}_{0}^{N}$.

- Symmetry. $\psi_{i}(N, v)=\psi_{j}(N, v)$ for all $(N, v) \in \mathcal{G}_{0}^{N}$ and $i, j \in N$ being symmetric in $(N, v)$.

- Dummy player property. $\psi_{i}(N, v)=v(\{i\})$ for all $(N, v) \in \mathcal{G}_{0}^{N}$ and $i \in N$ being a dummy player in $(N, v)$.

- Dummifying player property. $\psi_{i}(N, v)=v(\{i\})$ for all $(N, v) \in \mathcal{G}_{0}^{N}$ and $i \in N$ being a dummifying player in $(N, v)$.

- Weak linearity. For all $a \in \mathbb{R}$, and all $(N, v),(N, w) \in \mathcal{G}_{0}^{N}$ such that there exists $c \in \mathbb{R}_{+}$with $w(\{i\})=c v(\{i\})$ for all $i \in N$, if $(N, a v+w) \in$ $\mathcal{G}_{0}^{N}$, then $\psi(N, a v+w)=a \psi(N, v)+\psi(N, w)$.

The first three axioms are classical, except that they are defined on subclass $\mathcal{G}_{0}^{N}$. The dummifying player property, proposed by Casajus and Huettner (2014), states that a dummifying player just earns its own stand-alone worth. Weak linearity, proposed by Béal et al. (2018), states that when taking a linear combination of two games, where the ratio between the stand-alone 
worths is the same in both games, the payoff allocation equals the corresponding linear combination of the payoff vectors of the two separate games. This axiom is a weak version of the axiom of linearity as proposed by Shapley (1953). If $a=1$, then weak linearity reduces to weak additivity, which is introduced and studied in Besner (2019).

\section{Proportionality principle}

In this section, we introduce a new axiom, called balanced treatment, and characterize the proportional division value.

Definition 1. Players $i, j \in N, i \neq j$, are weak symmetric in $(N, v)$ if $v(S \cup\{i\})=v(S \cup\{j\})$ for all $S \subseteq N \backslash\{i, j\}, S \neq \emptyset$.

So, two players being weak symmetric still allows them to have a different singelton worth, but their contribution to any nonempty coalition of other players should be equal. Notice that in two-player games both players are always weak symmetric. We now introduce a proportionality property, comparable to symmetry, which says that the payoffs to two weak symmetric players are in the same proportion as their stand-alone worths. This axiom can be considered as a strengthening of Shapley's symmetry axiom in the sense that it implies that any two symmetric players in any game should earn the same payoff.

- Balanced treatment. $\frac{\psi_{i}(N, v)}{v(\{i\})}=\frac{\psi_{j}(N, v)}{v(\{j\})}$ for all $(N, v) \in \mathcal{G}_{0}^{N}$ and $i, j \in$ $N$ being a pair of weak symmetric players in $(N, v)$.

Next, we exactly characterize the class of values on $\mathcal{G}_{0}^{N}$ that satisfies efficiency, weak linearity, and balanced treatment.

Theorem 1. A value $\psi$ on $\mathcal{G}_{0}^{N}$ satisfies efficiency, weak linearity, and balanced treatment if and only if for each $(N, v) \in \mathcal{G}_{0}^{N}$ and each $i \in N$,

$\psi_{i}(N, v)=\frac{v(\{i\})}{\sum_{j \in N} v(\{j\})} v(N)+v(\{i\})\left[\sum_{\substack{S: i \in S \neq N \\|S| \geq 2}} \frac{\lambda_{S}}{\sum_{j \in S} v(\{j\})} v(S)-\sum_{\substack{S: i \notin S \\|S| \geq 2}} \frac{\lambda_{S}}{\sum_{j \in N \backslash S} v(\{j\})} v(S)\right]$, 
where $\lambda_{S}$ for each $S \subset N$ with $|S| \geq 2$ is a real number such that

$$
\frac{\lambda_{S}}{\sum_{j \in S} v(\{j\}) \sum_{j \in N \backslash S} v(\{j\})}=\frac{\lambda_{T}}{\sum_{j \in T} v(\{j\}) \sum_{j \in N \backslash T} v(\{j\})}, \quad \text { if }|S|=|T| .
$$

The lengthy proof of Theorem 1 is given in the Appendix. The proof uses the following proposition, which characterizes the PD value on the class of two-player games in $\mathcal{G}_{0}^{N}$. Since the two players in a two-player game $(N, v) \in$ $\mathcal{G}_{0}^{N}$ are always weak symmetric, if value $\psi$ satisfies balanced treatment, then $\frac{\psi_{i}(N, v)}{v(\{i\})}=\frac{\psi_{j}(N, v)}{v(\{j\})}$ for $i, j \in N$. By efficiency, we then obtain that the worth of the grand coalition is allocated proportional to the stand-alone worths. Since it is clear that the PD value satisfies these two axioms, we have the following proposition.

Proposition 1. Let $N \in \mathcal{N}$ with $n=2$. The $P D$ value is the unique value on $\mathcal{G}_{0}^{N}$ satisfying efficiency and balanced treatment.

The values characterized in Theorem 1, can be seen as modifications of the PD value, where to every game they first apply the PD value and then make a 'correction' that is based on the stand-alone worth of a player and the difference between weighted sums of the worths of all other coalitions with and without this player. The weights depend on all stand-alone worths. In this sense, (2) bears some similarity with the for the family of efficient, linear and symmetric (ELS) values (Lemma 9, Ruiz et al. 1998):

$$
\psi_{i}(N, v)=\frac{v(N)}{n}+\sum_{S: i \in S \neq N} \frac{\rho_{s}}{s} v(S)-\sum_{S: i \notin S} \frac{\rho_{s}}{n-s} v(S),
$$

where $\rho_{s}, s \in\{1,2, \ldots, n-1\}$, is a real number. The ELS values can be seen as first applying equal division and then make a correction based on a weighted sum of differences between worths of coalitions with and without a player. Specifically, if $v(\{i\})=v(\{j\})$ for all $i, j \in N$, (2) coincides with the above equation. 
Remark 1. Note that (3) indicates that all coefficients of coalitions of the same size are uniquely determined as soon as any one of them is given. For computational convenience, given $\left\{\lambda_{S} \in \mathbb{R}|S \subset N| S \mid, \geq 2\right\}$, denoting $\lambda_{s}=\frac{\lambda_{S}}{\sum_{j \in S} v(\{j\}) \sum_{j \in N \backslash S} v(\{j\})}$, 2p can be rewritten as

$\psi_{i}(N, v)=\frac{v(\{i\}) v(N)}{\sum_{j \in N} v(\{j\})}+v(\{i\})\left[\sum_{S: i \in S \neq N} \sum_{j \in N \backslash S} v(\{j\}) \lambda_{s} v(S)-\sum_{S: i \notin S} \sum_{j \in S} v(\{j\}) \lambda_{s} v(S)\right]$,

where $\lambda_{1}=0$, and $\lambda_{s}, s \in\{1,2, \ldots, n-1\}$, is a function with respect to $\lambda_{S}$ and all stand-alone worths. Since $\lambda_{s}$ might be different for different games which stand-alone worths are different, (4) cannot be directly used to verify weak linearity.

A next question is whether the class of values characterized in Theorem 1 contains a value that satisfies the dummy player property. It turns out that, for games with at least three players, the dummy player property is incompatible with the three axioms in Theorem 1.

Theorem 2. Let $N \in \mathcal{N}$ with $n \geq 3$. There is no value on $\mathcal{G}_{0}^{N}$ satisfying efficiency, weak linearity, balanced treatment, and the dummy player property.

Proof. Let $\psi$ be a value satisfying these axioms. First, suppose that $n \geq 4$. Consider any game $(N, v) \in \mathcal{G}_{0}^{N}$ and $i \in N$ such that $i$ is a dummy player in $(N, v)$. By Theorem 1, we have

$$
\begin{aligned}
\psi_{i}(N, v)= & \frac{v(\{i\})}{\sum_{j \in N} v(\{j\})} v(N)+\sum_{\substack{S: i \in S \neq N \\
|S| \geq 2}} \frac{v(\{i\}) \cdot \lambda_{S}}{\sum_{j \in S} v(\{j\})} v(S)-\sum_{\substack{S: i \notin S \\
|S| \geq 2}} \frac{v(\{i\}) \cdot \lambda_{S}}{\sum_{j \in N \backslash S} v(\{j\})} v(S) \\
= & \frac{v(\{i\}) v(N)}{\sum_{j \in N} v(\{j\})}+\sum_{\substack{S: i \notin S \\
1 \leq|S| \leq n-2}} \frac{v(\{i\}) \cdot \lambda_{S \cup\{i\}}}{\sum_{j \in S \cup\{i\}} v(\{j\})} v(S \cup\{i\})-\sum_{\substack{S: i \notin S \\
|S| \geq 2}} \frac{v(\{i\}) \cdot \lambda_{S}}{\sum_{j \in N \backslash S} v(\{j\})} v(S) \\
= & \frac{v(\{i\})[v(N \backslash\{i\})+v(\{i\})]}{\sum_{j \in N} v(\{j\})}+\sum_{j \in N \backslash\{i\}} \frac{v(\{i\}) \cdot \lambda_{\{i, j\}}}{v(\{i\})+v(\{j\})} v(\{i, j\})-\lambda_{N \backslash\{i\}} v(N \backslash\{i\}) \\
& +\sum_{\substack{S: i \notin S \\
2 \leq|S| \leq n-2}}\left[\frac{v(\{i\}) \cdot \lambda_{S \cup\{i\}}}{\sum_{j \in S \cup\{i\}} v(\{j\})} v(S \cup\{i\})-\frac{v(\{i\}) \cdot \lambda_{S}}{\sum_{j \in N \backslash S} v(\{j\})} v(S)\right]
\end{aligned}
$$




$$
\begin{aligned}
& =\frac{v(\{i\}) v(\{i\})}{\sum_{j \in N} v(\{j\})}+v(\{i\}) \sum_{j \in N \backslash\{i\}} \lambda_{\{i, j\}}+\left[\frac{v(\{i\})}{\sum_{j \in N} v(\{j\})}-\lambda_{N \backslash\{i\}}\right] v(N \backslash\{i\}) \\
& \quad+\sum_{\substack{S: i \notin S \\
2 \leq|S| \leq n-2}} \frac{v(\{i\}) \cdot \lambda_{S \cup\{i\}}}{\sum_{j \in S \cup\{i\}} v(\{j\})} v(\{i\})+\sum_{\substack{S: i \notin S \\
2 \leq|S| \leq n-2}}\left[\frac{v(\{i\}) \cdot \lambda_{S \cup\{i\}}}{\sum_{j \in S \cup\{i\}} v(\{j\})}-\frac{v(\{i\}) \cdot \lambda_{S}}{\sum_{j \in N \backslash S} v(\{j\})}\right] v(S),
\end{aligned}
$$

where the third equality follows from $i$ being a dummy player in $(N, v)$.

Since, by the dummy player property, the payoff of dummy player $i$ should not depend on $v(S), i \notin S$ and $2 \leq|S| \leq n-1$, the third term and the fifth term of the above equation must be equal to 0 , which yields

$$
\begin{aligned}
\lambda_{N \backslash\{i\}} & =\frac{v(\{i\})}{\sum_{j \in N} v(\{j\})}, \\
\frac{\lambda_{S \cup\{i\}}}{\lambda_{S}} & =\frac{\sum_{j \in S \cup\{i\}} v(\{j\})}{\sum_{j \in N \backslash S} v(\{j\})} \text { for } S \subset N \text { with } 2 \leq|S| \leq n-2 .
\end{aligned}
$$

Since $\lambda_{S}$ for each $S \subset N$ with $|S| \geq 2$ satisfies (3), then (5) and (3) together imply that

$$
\lambda_{N \backslash\left\{k_{1}\right\}}=\frac{v\left(\left\{k_{1}\right\}\right) \sum_{j \in N \backslash\left\{k_{1}\right\}} v(\{j\})}{\sum_{j \in N \backslash\{i\}} v(\{j\}) \sum_{j \in N} v(\{j\})} \quad \text { for any } k_{1} \in N \backslash\{i\} .
$$

By using (6), we have

$$
\begin{aligned}
\lambda_{N \backslash\left\{i, k_{1}\right\}} & =\frac{\sum_{j \in\left\{i, k_{1}\right\}} v(\{j\})}{\sum_{j \in N \backslash\left\{k_{1}\right\}} v(\{j\})} \lambda_{N \backslash\left\{k_{1}\right\}} \\
& =\frac{v\left(\left\{k_{1}\right\}\right) \sum_{j \in\left\{i, k_{1}\right\}} v(\{j\})}{\sum_{j \in N \backslash\{i\}} v(\{j\}) \sum_{j \in N} v(\{j\})} .
\end{aligned}
$$

The above equation together with (3) imply that, for any $k_{2} \in N \backslash\left\{i, k_{1}\right\}$,

$$
\begin{aligned}
\lambda_{N \backslash\left\{k_{1}, k_{2}\right\}} & =\frac{\sum_{j \in N \backslash\left\{k_{1}, k_{2}\right\}} v(\{j\}) \sum_{j \in\left\{k_{1}, k_{2}\right\}} v(\{j\})}{\sum_{j \in N \backslash\left\{i, k_{1}\right\}} v(\{j\}) \sum_{j \in\left\{i, k_{1}\right\}} v(\{j\})} \lambda_{N \backslash\left\{i, k_{1}\right\}} \\
& =\frac{\sum_{j \in N \backslash\left\{k_{1}, k_{2}\right\}} v(\{j\}) \sum_{j \in\left\{k_{1}, k_{2}\right\}} v(\{j\}) v\left(\left\{k_{1}\right\}\right)}{\sum_{j \in N \backslash\left\{i, k_{1}\right\}} v(\{j\}) \sum_{j \in N \backslash\{i\}} v(\{j\}) \sum_{j \in N} v(\{j\})} .
\end{aligned}
$$

Now, exchanging the order of $k_{1}$ and $k_{2}$, we have

$$
\lambda_{N \backslash\left\{k_{1}, k_{2}\right\}}=\frac{\sum_{j \in N \backslash\left\{k_{1}, k_{2}\right\}} v(\{j\}) \sum_{j \in\left\{k_{1}, k_{2}\right\}} v(\{j\}) v\left(\left\{k_{2}\right\}\right)}{\sum_{j \in N \backslash\left\{i, k_{2}\right\}} v(\{j\}) \sum_{j \in N \backslash\{i\}} v(\{j\}) \sum_{j \in N} v(\{j\})} .
$$


Therefore, it must be that

$$
\frac{v\left(\left\{k_{1}\right\}\right)}{\sum_{j \in N \backslash\left\{i, k_{1}\right\}} v(\{j\})}=\frac{v\left(\left\{k_{2}\right\}\right)}{\sum_{j \in N \backslash\left\{i, k_{2}\right\}} v(\{j\})},
$$

from which it follows that $v\left(\left\{k_{1}\right\}\right)=v\left(\left\{k_{2}\right\}\right)$ for any $k_{1}, k_{2} \in N \backslash\{i\}$. This contradicts the definition of $\mathcal{G}_{0}^{N}$.

Similarly, for $n=3$, we also have a contradiction to the fact that the stand-alone worths of players are not always the same.

Remark 2. Note that the contradiction vanishes if $n=2$. In fact, in this case, the PD value satisfies these axioms.

Since the PD value satisfies efficiency, weak linearity, and balanced treatment on $\mathcal{G}_{0}^{N}$, it belongs to the class of values characterized in Theorem 1. In fact, it is the value corresponding to $\lambda_{S}=0$ for all $S \subseteq N$. As it turns out, replacing the dummy player property in the impossibility theorem 2 by the dummifying player property, characterizes the PD value (also for two-player games).

Theorem 3. The PD value is the unique value on $\mathcal{G}_{0}^{N}$ that satisfies efficiency, weak linearity, balanced treatment, and the dummifying player property.

Proof. It is obvious that the PD value satisfies efficiency, weak linearity, balanced treatment, and the dummifying player property. It remains to prove the uniqueness part. Let $\psi$ be a value satisfying these axioms. By Theorem 1. any value satisfying efficiency, weak linearity and balanced treatment is given by (2) for some $\lambda_{S}(S \subseteq N,|S| \geq 2)$ satisfying (3). To achieve $\lambda_{S}$, we consider a modified game $\left(N, v_{i}\right) \in \mathcal{G}_{0}^{N}$ with respect to $(N, v) \in \mathcal{G}_{0}^{N}$ and $i \in N$, defined by

$$
v_{i}(S)= \begin{cases}v(\{j\}), & \text { if } S=\{j\} \text { for all } j \in N, \\ \sum_{j \in S} v(\{j\}), & \text { if } i \in S \text { and }|S| \geq 2, \\ v(S), & \text { otherwise. }\end{cases}
$$


Applying (2) to the game $\left(N, v_{i}\right)$, we have

$$
\psi_{i}\left(N, v_{i}\right)=v(\{i\})+v(\{i\})\left[\sum_{\substack{S: i \in S \neq N \\|S| \geq 2}} \lambda_{S}-\sum_{\substack{S: i \notin S \\|S| \geq 2}} \frac{\lambda_{S} v(S)}{\sum_{j \in N \backslash S} v(\{j\})}\right] .
$$

Since $i$ is dummifying in $\left(N, v_{i}\right)$, the dummifying player property requires that $\psi_{i}\left(N, v_{i}\right)=v(\{i\})$, and thus

$$
\sum_{\substack{S: i \in S \neq N \\|S| \geq 2}} \lambda_{S}-\sum_{\substack{S: i \notin S \\|S| \geq 2}} \frac{\lambda_{S} v(S)}{\sum_{j \in N \backslash S} v(\{j\})} \equiv 0 .
$$

It follows that

$$
\sum_{s=2}^{n-1}\left[\sum_{\substack{S: i \in S \\|S|=s}} \lambda_{S}-\sum_{\substack{S: i q S \\|S|=s}} \frac{\lambda_{S} v(S)}{\sum_{j \in N \backslash S} v(\{j\})}\right] \equiv 0 .
$$

We will show that $\lambda_{S}=0$ for all $S \subset N$ in (7). Suppose by contradiction that there exists some $S \subset N$ with $s \in\{2, \ldots, n-1\}$ such that $\lambda_{S} \neq 0$ and $|S|=s$. Let $\mathcal{S}=\left\{s_{1}, s_{2}, \ldots, s_{m}\right\}$ be the set of such coalitional sizes. Note that (3) implies that if $\lambda_{S} \neq 0$, then all coefficients of coalitions of the same size $s$ are not equal to zero. We denote by $\mathcal{S}_{k}=\left\{S_{k}^{1}, S_{k}^{2}, \ldots, S_{k}^{h}\right\}$, $k=1, \ldots, m, h=\left(\begin{array}{c}n \\ s_{k}\end{array}\right)$, the set of all coalitions of the same size $s_{k} \in \mathcal{S}$. Pick any $S_{k}^{r} \in \mathcal{S}_{k}$ with $i \in S_{k}^{r}$. By 33 , we have $\lambda_{S_{k}^{t}}=\frac{\sum_{j \in S_{k}^{t}} v(\{j\}) \sum_{j \in N \backslash S_{k}^{t}} v(\{j\})}{\sum_{j \in S_{k}^{r}} v(\{j\}) \sum_{j \in N \backslash S_{k}^{r}} v(\{j\})} \lambda_{S_{k}^{r}}$ for any $S_{k}^{t} \in \mathcal{S}_{k}$ (it obviously holds for the case $S_{k}^{t}=S_{k}^{r}$ ). With this equality, (7) can be written as

$$
\sum_{s_{k} \in \mathcal{S}}\left[A\left(\mathcal{S}_{k}\right)-\sum_{S_{k}^{t} \in \mathcal{S}_{k}, i \notin S_{k}^{t}} B\left(S_{k}^{t}\right) v\left(S_{k}^{t}\right)\right] \lambda_{S_{k}^{r}}=0,
$$

where $A\left(\mathcal{S}_{k}\right)=\sum_{S_{k}^{t} \in \mathcal{S}_{k}, i \in S_{k}^{t}} \frac{\sum_{j \in S_{k}^{t}} v(\{j\}) \sum_{j \in N \backslash S_{k}^{t}} v(\{j\})}{\sum_{j \in S_{k}^{r}} v(\{j\}) \sum_{j \in N \backslash S_{k}^{r}} v(\{j\})}$ and $B\left(S_{k}^{t}\right)=\frac{\sum_{j \in S_{k}^{t}} v(\{j\})}{\sum_{j \in S_{k}^{r}} v(\{j\}) \sum_{j \in N \backslash S_{k}^{r}} v(\{j\})}$.

Now, pick any $s_{l} \in\left\{s_{1}, s_{2}, \ldots, s_{m}\right\}$ and any $c \in \mathbb{R} \backslash\{0\}$, and consider the game $\left(N, v_{i, s_{l}}\right) \in \mathcal{G}_{0}^{N}$ given by

$$
v_{i, s_{l}}(S)= \begin{cases}v_{i}(S)+c, & \text { if }|S|=s_{l} \text { and } i \notin S, \\ v_{i}(S), & \text { otherwise. }\end{cases}
$$


Note that (3) shows that $\lambda_{S}$ only depends on the size of $S$ and the worths of all singleton coalitions. Therefore, since $i$ is a dummifying player in $\left(N, v_{i, s_{l}}\right)$, for the above game we can obtain an equation similar as (8) but with an additional term that depends on $c$,

$$
\sum_{s_{k} \in \mathcal{S}}\left[A\left(\mathcal{S}_{k}\right)-\sum_{S_{k}^{t} \in \mathcal{S}_{k}, i \notin S_{k}^{t}} B\left(S_{k}^{t}\right) v\left(S_{k}^{t}\right)\right] \lambda_{S_{k}^{r}}-c \lambda_{S_{l}^{r}} \sum_{S_{l}^{t} \in \mathcal{S}_{l}, i \notin S_{l}^{t}} B\left(S_{l}^{t}\right)=0 .
$$

Together with this equation and $(8)$, it holds that $-c \lambda_{S_{l}^{r}} \sum_{S_{l}^{t} \in \mathcal{S}_{l}, i \notin S_{l}^{t}} B\left(S_{l}^{t}\right)=$ 0 , yielding $\lambda_{S_{l}^{r}}=0$, which is a contradiction.

Remark 3. If the domain is restricted to the class containing all individually equal games (i.e., $(N, v) \in \mathcal{G}_{0}^{N}$ such that $v(\{i\})=v(\{j\})$ for all $\left.i, j \in N\right)$, then weak linearity and balanced treatment reduce to linearity and symmetry, respectively. Denoting this class of games by $\mathcal{G}_{e}^{N}$, in contrast to Theorem 2 and Theorem 3, one can obtain the following results: (i) The Shapley value is the unique value on $\mathcal{G}_{e}^{N}$ that satisfies efficiency, additivity, symmetry, and the dummy player property; (ii) The ED value is the unique value on $\mathcal{G}_{e}^{N}$ that satisfies efficiency, additivity, symmetry, and the dummifying player property.

Remark 4. We conclude this section by comparing our results with the main results in Casajus and Huettner (2014). Recall that Casajus and Huettner (2014) show that on the domain of TU-games $\mathcal{G}^{N}$, the equal surplus division value treats dummifying players in the same way as the Shapley value handles dummy players. Restricting ourselves to the subclass $\mathcal{G}_{0}^{N}$, notice that the $\mathrm{PD}$ value can be considered as a variation of the equal surplus division value since $P D_{i}(N, v)=v(\{i\})+\frac{v(\{i\})}{\sum_{j \in N} v(\{j\})}\left[v(N)-\sum_{j \in N} v(\{j\})\right]$. Interestingly, Theorem 3 gives a characterization of the $\mathrm{PD}$ value using the dummifying player property, whereas, for $|N| \geq 3$, using the dummy player property instead of the dummifying player property leads to an impossibility, as we saw in Theorem 2. 


\section{Monotonicity}

In this section, we present axiomatic characterizations of the proportional division value by imposing three appropriate monotonicity axioms being weaker versions of classical monotonicity axioms in the literature.

- Weak coalitional surplus equivalence 4 . For all $(N, v),(N, w) \in \mathcal{G}_{0}^{N}$ with $v(\{j\})=w(\{j\})$ for all $j \in N$, and $i \in N$ being a dummifying player in $(N, w)$, we have $\psi_{i}(N, v+w)=\psi_{i}(N, v)+w(\{i\})$.

- Weak coalitional surplus monotonicity. For all $(N, v),(N, w) \in$ $\mathcal{G}_{0}^{N}$ with $w(\{j\})=c v(\{j\})$ for all $j \in N$ and $c \in \mathbb{R}_{+}$, and $i \in N$ such that $v(S)-\sum_{j \in S} v(\{j\}) \geq w(S)-\sum_{j \in S} w(\{j\})$ for all $S \subseteq N$ with $i \in S$, we have $\psi_{i}(N, v)-v(\{i\}) \geq \psi_{i}(N, w)-w(\{i\})$.

- Weak coalitional monotonicity. For all $(N, v),(N, w) \in \mathcal{G}_{0}^{N}$ with $v(\{j\})=w(\{j\})$ for all $j \in N$, and $i \in N$ such that $v(S) \geq w(S)$ for all $S \subseteq N$ with $i \in S$, we have $\psi_{i}(N, v) \geq \psi_{i}(N, w)$.

Weak coalitional surplus equivalence states that the payoff of a player increases with its stand-alone worth if we add a game in which this player is a dummifying player and each stand-alone worth is the same as that of the original game.

Weak coalitional surplus monotonicity states that if two games in which the stand-alone worths of all players are in the same proportion to each other and the surplus of every coalition a player belongs to (measured by the worth of the coalition minus the sum of the stand-alone worths of its players) weakly increases, then the relative payoff of this player (being the difference between the payoff and stand-alone worth) should not decrease.

\footnotetext{
${ }^{4}$ Weak coalitional surplus equivalence is a monotonicity principle due to the fact that it is implied by weak coalitional surplus monotonicity with $c=2$. See Lemma 1 .
} 
Weak coalitional monotonicity states that the payoff of a player should not decrease whenever the worth of every coalition containing this player weakly increases, while the worth of every singleton coalition remains unchanged.

Weak coalitional surplus equivalence (respectively, weak coalitional surplus monotonicity) is a weak version of coalitional surplus equivalence $\mathrm{f}^{5}$ (respectively, coalitional surplus monotonicity ${ }^{6}$ as defined in Casajus and Huettner (2014). Weak coalitional monotonicity is stronger than coalitional monotonicity ${ }^{7}$ as defined in Shubik (1962), while it is weaker than coalitional monotonicity ${ }^{8}$ as defined in van den Brink (2007).

Notice that weak coalitional monotonicity is a specific case of weak coalitional surplus monotonicity taking $c=1$. On the other hand, weak coalitional surplus monotonicity implies weak coalitional surplus equivalence.

Lemma 1. Every value on $\mathcal{G}_{0}^{N}$ that satisfies weak coalitional surplus monotonicity also satisfies weak coalitional surplus equivalence.

Proof. Suppose that value $\psi$ satisfies weak coalitional surplus monotonicity. Consider a pair of games $(N, v),(N, v+w) \in \mathcal{G}_{0}^{N}$, where $v(\{j\})=w(\{j\})$ for all $j \in N$, and $i \in N$ such that $w(S)=\sum_{j \in S} w(\{j\})$ for all $S \subseteq N$ with $i \in S$. Since $(v+w)(S)-\sum_{j \in S}(v+w)(\{j\})=v(S)-\sum_{j \in S} v(\{j\})$ for all $S \subseteq N$ with $i \in S$, by weak coalitional surplus monotonicity we have $\psi_{i}(N, v+w)-(v+w)(\{i\})=\psi_{i}(N, v)-v(\{i\})$. It follows that $\psi_{i}(N, v+$

\footnotetext{
${ }^{5} \mathrm{~A}$ value $\psi$ satisfies coalitional surplus equivalence if $\psi_{i}(N, v+w)=\psi_{i}(N, v)+w(\{i\})$ for all $(N, v),(N, w) \in \mathcal{G}^{N}$ and $i \in N$ being a dummifying player in $(N, w)$.

${ }^{6} \mathrm{~A}$ value $\psi$ satisfies coalitional surplus monotonicity if $\psi_{i}(N, v)-v(\{i\}) \geq \psi_{i}(N, w)-$ $w(\{i\})$ for all $(N, v),(N, w) \in \mathcal{G}^{N}$, and $i \in N$ such that $v(S)-\sum_{j \in S} v(\{j\}) \geq w(S)-$ $\sum_{j \in S} w(\{j\})$ for all $S \subseteq N$ with $i \in S$.

${ }^{7} \mathrm{~A}$ value $\psi$ satisfies Shubik's version of coalitional monotonicity if $\psi_{i}(N, v) \geq \psi_{i}(N, w)$ for all $(N, v),(N, w) \in \mathcal{G}^{N}$ and $i \in N$ such that $v(S) \geq w(S)$ for all $S \subseteq N$ with $i \in S$, and $v(S)=w(S)$ for all $S \subseteq N \backslash\{i\}$.

${ }^{8}$ A value $\psi$ satisfies van den Brink's version of coalitional monotonicity if $\psi_{i}(N, v) \geq$ $\psi_{i}(N, w)$ for all $(N, v),(N, w) \in \mathcal{G}^{N}$ and $i \in N$ such that $v(S) \geq w(S)$ for all $S \subseteq N$ with $i \in S$.
} 
$w)=\psi_{i}(N, v)+w(\{i\})$, which shows that $\psi$ satisfies weak coalitional surplus equivalence.

Considering weak coalitional surplus equivalence and weak coalitional surplus monotonicity, the PD value is characterized by either one of these axioms in addition to efficiency and balanced treatment.

Theorem 4. (i) The PD value is the unique value on $\mathcal{G}_{0}^{N}$ that satisfies efficiency, balanced treatment, and weak coalitional surplus equivalence.

(ii) The $P D$ value is the unique value on $\mathcal{G}_{0}^{N}$ that satisfies efficiency, balanced treatment, and weak coalitional surplus monotonicity.

Proof. (i) It is clear that the PD value satisfies efficiency, balanced treatment, and weak coalitional surplus equivalence. Now let $\psi$ be a value on $\mathcal{G}_{0}^{N}$ satisfying the three axioms. For $n=1$, (1) is satisfied by efficiency. For $n=2$, (1) is obtained from Proposition 11. For $n \geq 3$, uniqueness follows by induction on $d(v)=\mid\left\{T \subseteq N \mid v(T)-\frac{1}{2} \sum_{j \in T} v(\{j\}) \neq 0\right.$ and $\left.|T| \geq 2\right\} \mid$.

Intialization. If $d(v)=0$ then $v(N)=\frac{1}{2} \sum_{j \in N} v(\{j\})$. For any $(N, v) \in$ $\mathcal{G}_{0}^{N}$, define $\left(N, v^{0}\right) \in \mathcal{G}_{0}^{N}$ as follows:

$$
v^{0}(T)=v(T)-\frac{1}{2} \sum_{j \in T} v(\{j\}) \text { for all } T \subseteq N .
$$

Notice that, by $d(v)=0, v^{0}(T)=0$ for all $T \subseteq N$ with $|T| \geq 2$. Clearly, in this case, all players $i, j \in N$ are weak symmetric in $\left(N, v^{0}\right)$ and $v^{0}(N)=0$. By efficiency and balanced treatment, we have $\psi_{i}\left(N, v^{0}\right)=0$ for all $i \in N$. Notice that $\left(v-v^{0}\right)(\{i\})=v(\{i\})-v(\{i\})+\frac{1}{2} v(\{i\})=\frac{1}{2} v(\{i\})$ for all $i \in N$, and all players are dummifying in $\left(N, v-v^{0}\right)$ since $\left(v-v^{0}\right)(T)=v(T)-v(T)+$ $\frac{1}{2} \sum_{j \in T} v(\{j\})=\frac{1}{2} \sum_{j \in T} v(\{j\})=\sum_{j \in T}\left(v-v^{0}\right)(\{j\})$. It follows from weak coalitional surplus equivalence that $\psi_{i}(N, v)=\psi_{i}\left(N, v^{0}\right)+\psi_{i}\left(N, v-v^{0}\right)=$ $\psi_{i}\left(N, v^{0}\right)+\frac{1}{2} v(\{i\})$ for all $i \in N$. Thus, we obtain $\psi_{i}(N, v)=\frac{1}{2} v(\{i\})=$ $P D_{i}(N, v)$ for all $i \in N$.

Proceeding by induction, assume that $\psi_{i}(N, w)=P D_{i}(N, w)$ if $d(w)<$ $d(v)$. Let $\mathcal{S}=\left\{S_{1}, S_{2}, \ldots, S_{d(v)}\right\}$ be the set of coalitions such that $v\left(S_{k}\right)-$ 
$\frac{1}{2} \sum_{j \in S_{k}} v(\{j\}) \neq 0$ and $\left|S_{k}\right| \geq 2$. Let $S$ be the intersection of all such coalitions $S_{k}$, i.e., $S=\bigcap_{1 \leq k \leq d(v)} S_{k}$. We distinguish between two cases:

Case (a): $i \in N \backslash S$. Each player $i \in N \backslash S$ is a member of at most $d(v)-1$ coalitions in $\mathcal{S}$, and at least one $S_{i} \in \mathcal{S}$ such that $i \notin S_{i}$ (obviously, $S_{i} \neq N$ ). For $(N, v) \in \mathcal{G}_{0}^{N}$, define two associated games as follows:

$$
\begin{aligned}
& v^{i, 1}(T)= \begin{cases}v(T)-\frac{1}{2} \sum_{j \in T} v(\{j\}), & \text { if } T \neq S_{i}, \\
0, & \text { if } T=S_{i} .\end{cases} \\
& v^{i, 2}(T)= \begin{cases}\frac{1}{2} \sum_{j \in T} v(\{j\}), & \text { if } T \neq S_{i}, \\
v\left(S_{i}\right), & \text { if } T=S_{i} .\end{cases}
\end{aligned}
$$

Clearly, $v=v^{i, 1}+v^{i, 2}, v^{i, 1}(\{j\})=v^{i, 2}(\{j\})=\frac{1}{2} v(\{j\})$ for all $j \in N$, $d\left(v^{i, 1}\right)=d(v)-1$, and all players $i \in N \backslash S_{i}$ are dummifying in $\left(N, v^{i, 2}\right)$. Weak coalitional surplus equivalence and the induction hypothesis then imply that $\psi_{i}(N, v)=\psi_{i}\left(N, v^{i, 1}\right)+\frac{1}{2} v(\{i\})=P D_{i}\left(N, v^{i, 1}\right)+\frac{1}{2} v(\{i\})=$ $\frac{v^{i, 1}(\{i\})}{\sum_{j \in N} v^{i, 1}(\{j\})} v^{i, 1}(N)+\frac{1}{2} v(\{i\})=\frac{v(\{i\})}{\sum_{j \in N} v(\{j\})}\left[v(N)-\frac{1}{2} \sum_{j \in N} v(\{j\})\right]+\frac{1}{2} v(\{i\})=$ $\frac{v(\{i\})}{\sum_{j \in N} v(\{j\})} v(N)=P D_{i}(N, v)$ for all $i \in N \backslash S_{i}$. Since there exists such a $S_{i}$ for all $i \in N \backslash S$, we obtain $\psi_{i}(N, v)=P D_{i}(N, v)$ for all $i \in N \backslash S$.

Case (b): $i \in S$. If $S=\{i\}$, we obtain, by efficiency of $\psi$ and $P D$ and case (a), $\psi_{i}(N, v)=P D_{i}(N, v)$. If $|S| \geq 2$, each player $j \in S$ is a member of all coalitions in $\mathcal{S}$. Now we consider the game $\left(N, v^{0}\right)$ as defined by (9) in case $d(v)=0$. Clearly, here all players $j \in S$ are weak symmetric in $\left(N, v^{0}\right)$. By efficiency and balanced treatment of $\psi$ and case (a), we obtain that, for any $i \in S, \sum_{j \in S} \psi_{j}(N, v)=\sum_{j \in S} \frac{v(\{j\})}{v(\{i\})} \psi_{i}(N, v)=v(N)-$ $\sum_{j \in N \backslash S} \psi_{j}(N, v)=v(N)-\sum_{j \in N \backslash S} P D_{i}(N, v)=\sum_{j \in S} \frac{v(\{j\})}{\sum_{k \in N} v(\{k\})} v(N)$, which yields $\psi_{i}(N, v)=P D_{i}(N, v)$.

The proof of (i) is complete. 
(ii) Since it is obvious that the PD value satisfies efficiency and balanced treatment, we only show that the PD value satisfies weak coalitional surplus monotonicity. Clearly, $w(\{j\})=c v(\{j\})$ for all $j \in N$ and $v(S)-$ $\sum_{j \in S} v(\{j\}) \geq w(S)-\sum_{j \in S} w(\{j\})$ for all $S \subseteq N$ with $i \in S$, imply that $v(N) \geq w(N)-\sum_{j \in N} w(\{j\})+\sum_{j \in N} v(\{j\})=w(N)-\sum_{j \in N} w(\{j\})+$ $\sum_{j \in N} \frac{w(\{j\})}{c}=w(N)-\left(1-\frac{1}{c}\right) \sum_{j \in N} w(\{j\})$. Thus, $P D_{i}(N, v)=\frac{v(\{i\})}{\sum_{j \in N} v(\{j\})} v(N)=$ $\frac{w(\{i\})}{\sum_{j \in N} w(\{j\})} v(N) \geq \frac{w(\{i\})}{\sum_{j \in N} w(\{j\})}\left[w(N)-\left(1-\frac{1}{c}\right) \sum_{j \in N} w(\{j\})\right]=P D_{i}(N, w)-$ $w(\{i\})+\frac{1}{c} w(\{i\})=P D_{i}(N, w)-w(\{i\})+v(\{i\})$.

Uniqueness follows from Theorem 4 and Lemma 1.

The next lemma shows a logical implication between the axioms in Theorem 3 and Theorem 4 (i), which implies that weak linearity in Theorem 3 can be weakened as weak additivity.

Lemma 2. Weak additivity and the dummifying player property together imply weak coalitional surplus equivalence.

Proof. Let $(N, v),(N, w) \in \mathcal{G}_{0}^{N}$ be two games such that $v(\{j\})=w(\{j\})$ for all $j \in N$, and $i \in N$ is dummifying in $(N, w)$. The dummifying player property implies that $\psi_{i}(N, w)=w(\{i\})$. Then weak additivity implies that $\psi_{i}(N, v+w)=\psi_{i}(N, v)+\psi_{i}(N, w)=\psi_{i}(N, v)+w(\{i\})$, as desired.

It is easy to verify that the PD value satisfies weak coalitional monotonicity. Interestingly, the PD value is characterized by replacing weak coalitional surplus monotonicity with weak coalitional monotonicity in Theorem 4 (ii). In this case, balanced treatment even can be weakened by requiring the proportionality only for games in which all players are weak symmetric.

- Weak balanced treatment. $\frac{\psi_{i}(N, v)}{v(\{i\})}=\frac{\psi_{j}(N, v)}{v(\{j\})}$ for all $(N, v) \in \mathcal{G}_{0}^{N}$ and $i, j \in N$ if all players are weak symmetric in $(N, v)$.

Theorem 5. The PD value is the unique value on $\mathcal{G}_{0}^{N}$ that satisfies efficiency, weak balanced treatment, and weak coalitional monotonicity. 
Proof. It is clear that the PD value satisfies efficiency, weak balanced treatment, and weak coalitional monotonicity. To show uniqueness, suppose that $\psi$ is a value satisfying the three axioms. For any game $(N, v) \in \mathcal{G}_{0}^{N}$, define the game $(N, w)$ by

$$
w(S)= \begin{cases}v(N), & \text { if } S=N, \\ v(\{j\}), & \text { if } S=\{j\} \text { for all } j \in N, \\ \min _{T \subseteq N,|T| \geq 2} v(T), & \text { otherwise. }\end{cases}
$$

Efficiency and weak balanced treatment imply that $\psi_{i}(N, w)=\frac{v(\{i\})}{\sum_{j \in N} v(\{j\})} v(N)$ for all $i \in N$. Pick any $i \in N$. Since $v(S) \geq w(S)$ for all $S \subseteq N$ with $i \in S$, then weak coalitional monotonicity implies that $\psi_{i}(N, v) \geq \psi_{i}(N, w)=$ $\frac{v(\{i\})}{\sum_{j \in N} v(\{j\})} v(N)$. Efficiency then implies that $\psi_{i}(N, v)=\frac{v(\{i\})}{\sum_{j \in N} v(\{j\})} v(N)$ for all $i \in N$.

Notice that by using the monotonicity axioms in Theorems 4 and 5 , we can get rid of weak linearity.

Considering the relationship between our monotonicity axioms and the stronger versions introduced in Casajus and Huettner (2014) and van den Brink (2007) (to characterize the ESD value or the ED value), from Theorems 45and 5, we obtain the following corollary.

Corollary 1 . Let $n \geq 2$. There is no value on $\mathcal{G}_{0}^{N}$ satisfying

(i) efficiency, balanced treatment, and coalitional surplus equivalence.

(ii) efficiency, balanced treatment, and coalitional surplus monotonicity.

(iii) efficiency, weak balanced treatment, and coalitional monotonicity.

As shown before, weak coalitional surplus monotonicity is stronger than both weak coalitional surplus equivalence and weak coalitional monotonicity. We conclude this section by mentioning two values to show logical independence of weak coalitional surplus equivalence and weak coalitional monotonicity. The value $\psi_{i}(N, v)=v(\{i\})-\frac{1}{n}\left[v(N)-\sum_{j \in N} v(\{j\})\right], i \in N$, satisfies 
weak coalitional surplus equivalence, but not weak coalitional monotonicity; the ED value $\psi_{i}(N, v)=\frac{v(N)}{n}, i \in N$, satisfies weak coalitional monotonicity, but not weak coalitional surplus equivalence.

\section{Consistency}

In this section, we consider a variable player set, and characterize the PD value by proportional standardness used in Ortmann (2000) and Khmelnitskaya and Driessen (2003), and projection consistency due to van den Brink and Funaki (2009) and van den Brink et al. (2016).

If a player $j \in N$ leaves game $(N, v)$ with a certain payoff, then the projection reduced game is a game on the remaining player set that assigns to every proper subset of $N \backslash\{j\}$ its worth in the original game, and to coalition $N \backslash\{j\}$ assigns its worth in $(N, v)$ minus the payoff assigned to player $j$.

Definition 2. Given a game $(N, v) \in \mathcal{G}_{0}$ with $|N| \geq 2$, a player $j \in N$ and a payoff vector $x \in \mathbb{R}^{N}$, the projection reduced game with respect to $j$ and $x$ is the game $\left(N \backslash\{j\}, v^{x}\right)$ given by

$$
v^{x}(S)= \begin{cases}v(N)-x_{j} & \text { if } S=N \backslash\{j\}, \\ v(S) & \text { if } S \subset N \backslash\{j\} .\end{cases}
$$

Projection consistency requires that the payoffs assigned to the remaining players in $N \backslash\{j\}$, after player $j$ leaving the game with its payoff according to a value $\psi$, is the same in the reduced game as in the original game.

Definition 3. A value $\psi$ satisfies projection consistency if for every game $(N, v) \in \mathcal{G}_{0}$ with $|N| \geq 3, j \in N$, and $x=\psi(N, v)$, it holds that $\left(N \backslash\{j\}, v^{x}\right) \in \mathcal{G}_{0}$, and $\psi_{i}\left(N \backslash\{j\}, v^{x}\right)=\psi_{i}(N, v)$ for all $i \in N \backslash\{j\}$.

Proportional standardness requires that in two player games we allocate the worth of the grand coalition over the two players proportional to their singleton worths. 
Definition 4. A value $\psi$ satisfies proportional standardness if for every game $(N, v) \in \mathcal{G}_{0}$ with $|N|=2$, it holds that

$$
\psi_{i}(N, v)=v(\{i\})+\frac{v(\{i\})}{v(\{i\})+v(\{j\})}[v(N)-v(\{i\})-v(\{j\})], \quad \forall i, j \in N .
$$

Proportional standardness is called proportional for two person games in Ortmann (2000).

Proposition 2. The PD value satisfies projection consistency on the class of all games $\mathcal{G}_{0}$.

Proof. For every $(N, v) \in \mathcal{G}_{0}$ with $|N| \geq 3$ and any $j \in N,\left(N \backslash\{j\}, v^{x}\right) \in \mathcal{G}_{0}$. For $x=P D(N, v)$ and $i \in N \backslash\{j\}$, we have

$$
\begin{aligned}
P D_{i}\left(N \backslash\{j\}, v^{x}\right) & =\frac{v^{x}(\{i\})}{\sum_{k \in N \backslash\{j\}} v^{x}(\{k\})} v^{x}(N \backslash\{j\}) \\
& =\frac{v(\{i\})}{\sum_{k \in N \backslash\{j\}} v(\{k\})}\left[v(N)-P D_{j}(N, v)\right] \\
& =\frac{v(\{i\})}{\sum_{k \in N \backslash\{j\}} v(\{k\})}\left[v(N)-\frac{v(\{j\})}{\sum_{k \in N} v(\{k\})} v(N)\right] \\
& =\frac{v(\{i\})}{\sum_{k \in N} v(\{k\})} v(N) \\
& =P D_{i}(N, v) .
\end{aligned}
$$

Projection consistency together with proportional standardness for twoplayer games characterizes the PD value on the class of games with at least two players. We denote the class of games in $\mathcal{G}_{0}$ with at least two players by $\hat{\mathcal{G}_{0}}$.

\footnotetext{
${ }^{9}$ Notice that, if $(N, v) \in \mathcal{G}_{0}$ with $|N|=2$ and $v(N)=0$, then for $x=P D(N, v)$, we have that $x_{i}=x_{j}=0$, and thus $\left(N \backslash\{j\}, v^{x}\right) \notin \mathcal{G}_{0}$ for any $j \in N$. In case $v(N) \neq 0$, for $x=P D(N, v)$, we have $\left(N \backslash\{j\}, v^{x}\right) \in \mathcal{G}_{0}$, since $\left[v(\{i\})>0 \Rightarrow P D_{j}(N, v)<v(N) \Rightarrow\right.$ $\left.v^{x}(\{i\})>0\right]($ similar if $v(\{i\})<0)$.
} 
Theorem 6. A value $\psi$ satisfies proportional standardness and projection consistency on the class of all games $\hat{\mathcal{G}}_{0}$ if and only if $\psi=P D$.

Proof. It is straightforward to show that the PD value satisfies proportional standardness. Projection consistency follows from Proposition 2. To show the 'only if' part, suppose that $\psi$ is a value satisfying proportional standardness and projection consistency.

If $|N|=2$, then $\psi(N, v)=P D(N, v)$ follows from proportional standardness.

Proceeding by induction, for $|N| \geq 3$, suppose that $\psi\left(N^{\prime}, w\right)=P D\left(N^{\prime}, w\right)$ whenever $\left|N^{\prime}\right|=|N|-1$. Take any $i, j \in N$ such that $i \neq j$. Let $x=\psi(N, v)$ and $y=P D(N, v)$. For the two reduced games $\left(N \backslash\{j\}, v^{x}\right)$ and $\left(N \backslash\{j\}, v^{y}\right)$, by the induction hypothesis, we have

$$
\begin{aligned}
x_{i}-y_{i} & =\psi_{i}\left(N \backslash\{j\}, v^{x}\right)-P D_{i}\left(N \backslash\{j\}, v^{y}\right) \\
& =P D_{i}\left(N \backslash\{j\}, v^{x}\right)-P D_{i}\left(N \backslash\{j\}, v^{y}\right) .
\end{aligned}
$$

By definition of the PD value and the projection reduced game, we have

$$
\begin{aligned}
& P D_{i}\left(N \backslash\{j\}, v^{x}\right)-P D_{i}\left(N \backslash\{j\}, v^{y}\right) \\
= & \frac{v^{x}(\{i\})}{\sum_{k \in N \backslash\{j\}} v^{x}(\{k\})}\left(v(N)-x_{j}\right)-\frac{v^{y}(\{i\})}{\sum_{k \in N \backslash\{j\}} v^{y}(\{k\})}\left(v(N)-y_{j}\right) \\
= & \frac{v(\{i\})}{\sum_{k \in N \backslash\{j\}} v(\{k\})}\left(y_{j}-x_{j}\right) .
\end{aligned}
$$

Together with (10), this implies that, for all $i, j \in N$ with $i \neq j$,

$$
x_{i}-y_{i}=\frac{v(\{i\})}{\sum_{k \in N \backslash\{j\}} v(\{k\})}\left(y_{j}-x_{j}\right) .
$$

Summing (11) over all $i \in N \backslash\{j\}$ yields

$$
\sum_{i \in N \backslash\{j\}}\left(x_{i}-y_{i}\right)=\frac{\sum_{i \in N \backslash\{j\}} v(\{i\})}{\sum_{k \in N \backslash\{j\}} v(\{k\})}\left(y_{j}-x_{j}\right)=y_{j}-x_{j} .
$$


On the other hand, (11) can be written as $v(\{i\})\left(y_{j}-x_{j}\right)=\sum_{k \in N \backslash\{j\}} v(\{k\})\left(x_{i}-\right.$ $\left.y_{i}\right)$. Summing this equality over all $j \in N \backslash\{i\}$, we have

$$
\begin{aligned}
& \sum_{j \in N \backslash\{i\}} \sum_{k \in N \backslash\{j\}} v(\{k\})\left(x_{i}-y_{i}\right)=\sum_{j \in N \backslash\{i\}} v(\{i\})\left(y_{j}-x_{j}\right) \\
\Leftrightarrow & \sum_{j \in N \backslash\{i\}}\left(v(\{i\})\left(x_{i}-y_{i}\right)+\sum_{k \in N \backslash\{i, j\}} v(\{k\})\left(x_{i}-y_{i}\right)\right)=v(\{i\}) \sum_{j \in N \backslash\{i\}}\left(y_{j}-x_{j}\right) \\
\Leftrightarrow & {\left[(n-1) v(\{i\})+(n-2) \sum_{j \in N \backslash\{i\}} v(\{j\})\right]\left(x_{i}-y_{i}\right)=v(\{i\}) \sum_{j \in N \backslash\{i\}}\left(y_{j}-x_{j}\right) . }
\end{aligned}
$$

Together with (12) and (13), it holds that $(n-2)\left(x_{i}-y_{i}\right) \sum_{j \in N} v(\{j\})=0$. Thus, $x_{i}-y_{i}=0$ for all $i \in N$. This shows that $\psi(N, v)=P D(N, v)$.

Proposition 1 and Theorem 6 together imply the following corollary.

Corollary 2. The PD value is the unique value on $\hat{\mathcal{G}}_{0}$ that satisfies efficiency, balanced treatment, and projection consistency.

\section{Proportional standardness: characterizations for two-player games}

In the previous section we imposed proportional standardness to characterize the PD value for any player set. Note that proportional standardness, as other two-player standardness axiom, is a quite strong axiom since it coincides with the definition of the PD value for two-player games. In this section, we support proportional standardness by showing how the PD value can be characterized on the class of two-player games. We first characterize the $\mathrm{PD}$ value for rational numbers, and then apply continuity to obtain a characterization for real worths. Denote $\mathcal{G}_{0}^{2}=\left\{(N, v) \in \mathcal{G}_{0}|| N \mid=2\right\}$ and $\mathcal{G}_{0 \mathbb{Q}}^{2}=\left\{(N, v) \in \mathcal{G}_{0}^{2} \mid v(S) \in \mathbb{Q}\right.$ for all $\left.S \subseteq N\right\}$, so the worths of coalitions in games in $\mathcal{G}_{0 \mathbb{Q}}^{2}$ are rational numbers.

First, we introduce two additional axioms, the first on $\mathcal{G}_{0 \mathbb{Q}}^{2}$ and the second on $\mathcal{G}_{0}^{2}$. 
- Grand worth additivity. For games $(N, v),(N, w) \in \mathcal{G}_{0 \mathbb{Q}}^{2}$ with $N=$ $\{i, j\}$ such that $v(\{i\})=w(\{i\})$ and $v(\{j\})=w(\{j\})$, it holds that $\psi(N, v)+\psi(N, w)=\psi(N, v \oplus w)$, where $(N, v \oplus w)$ is defined as: $(v \oplus w)(\{i\})=v(\{i\}),(v \oplus w)(\{j\})=v(\{j\})$ and $(v \oplus w)(N)=$ $v(N)+w(N)$.

- Inessential game property. For every game $(N, v) \in \mathcal{G}_{0}^{2}$ with $N=$ $\{i, j\}$ such that $v(\{i\})+v(\{j\})=v(\{i, j\})$, it holds that $\psi_{i}(N, v)=$ $v(\{i\})$ and $\psi_{j}(N, v)=v(\{j\})$.

Grand worth additivity ${ }^{10}$ states that for two games in which all worths are rational numbers and the stand-alone worths are the same, we consider the game where the stand-alone worths are the same as in the original game, and the worth of the grand coalition equals the sum of the worth of the grand coalition in the two games, then the payoff to each player equals the sum of the payoffs in the two separate games. The inessential game property is a well-known axiom requiring that players earn their stand-alone payoff in an inessential game. First, we show that these two axioms characterize the PD value on the class of two-player games with rational worths.

Proposition 3. A value $\psi$ on $\mathcal{G}_{0 \mathbb{Q}}^{2}$ satisfies grand worth additivity and the inessential game property if and only if $\psi=P D$.

Proof. It is obvious that $P D$ satisfies grand worth additivity and the inessential game property. To show uniqueness, suppose that $\psi$ is a value on $\mathcal{G}_{0 \mathbb{Q}}^{2}$ satisfying the two axioms. Let $(N, v) \in \mathcal{G}_{0 \mathbb{Q}}^{2}$ be an arbitrary game with $N=$ $\{i, j\}$. For any $\alpha \in \mathbb{Q}$, let the game $\left(N, v^{\alpha}\right)$ be defined by $v^{\alpha}(\{i\})=v(\{i\})$, $v^{\alpha}(\{j\})=v(\{j\})$ and $v^{\alpha}(N)=\alpha v(N)$. Clearly, $\left(N, v^{\alpha}\right) \in \mathcal{G}_{0 \mathbb{Q}}^{2}$.

If $\alpha=0$ then grand worth additivity implies that $\psi\left(N, v^{\alpha}\right)=0$. For any $\alpha \in \mathbb{Z}_{+} \prod$, since $\left(N, v^{\alpha}\right)=\left(N, v^{\alpha-1} \oplus v\right)=\cdots=(N, \underbrace{v \oplus \cdots \oplus v}_{\alpha})$,

${ }^{10}$ This axiom is similar to additivity in Moulin (1987) and Chun (1988).

${ }^{11} \mathbb{Z}, \mathbb{Z}_{+}$and $\mathbb{Z}_{-}$denote the sets of integers, positive integers and negative integers, 
grand worth additivity implies $\psi\left(N, v^{\alpha}\right)=\alpha \psi(N, v)$. For any $\alpha \in \mathbb{Z}_{-}$, since $(N, v^{\alpha} \oplus \underbrace{v \oplus \cdots \oplus v}_{|\alpha|})=\left(N, v^{0}\right)$, grand worth additivity and $\psi\left(N, v^{0}\right)=0$ (from above) imply $\psi\left(N, v^{\alpha}\right)=-|\alpha| \psi(N, v)+\psi\left(N, v^{0}\right)=\alpha \psi(N, v)$. Similarly, considering $(N, v)$, for any $\alpha \in \mathbb{Z}_{+},(N, v)=(N, \underbrace{v^{\frac{1}{\alpha}} \oplus \cdots \oplus v^{\frac{1}{\alpha}}}_{\alpha})$ implies that $\psi(N, v)=\alpha \psi\left(N, v^{\frac{1}{\alpha}}\right)$; for any $\alpha \in \mathbb{Z}_{-},(N, v \oplus \underbrace{v^{\frac{1}{\alpha}} \oplus \cdots \oplus v^{\frac{1}{\alpha}}}_{|\alpha|})=\left(N, v^{0}\right)$ implies that $\psi(N, v)=\alpha \psi\left(N, v^{\frac{1}{\alpha}}\right)$.

Next, take any $\alpha \in \mathbb{Q}$ and consider the game $\left(N, v^{\alpha}\right)$. Since any rational number can be written as a fraction, we suppose that $\alpha=\frac{k}{m}$ with $k \in \mathbb{Z}$ and $m \in \mathbb{Z} \backslash\{0\}$. Therefore, $\psi\left(N, v^{\alpha}\right)=\psi\left(N, v^{\frac{k}{m}}\right)=k \psi\left(N, v^{\frac{1}{m}}\right)=\frac{k}{m} \psi(N, v)=$ $\alpha \psi(N, v)$.

Take any game $(N, v) \in \mathcal{G}_{0 \mathbb{Q}}^{2}$. Taking $\alpha=\frac{v(\{i\})+v(\{j\})}{v(N)}, v^{\alpha}$ is an inessential game, and thus by the inessential game property, $\psi_{i}\left(N, v^{\alpha}\right)=v^{\alpha}(\{i\})=$ $v(\{i\})$. Since $\psi\left(N, v^{\alpha}\right)=\alpha \psi(N, v)$, we have $\psi_{i}(N, v)=\frac{1}{\alpha} \psi_{i}\left(N, v^{\alpha}\right)=$ $\frac{v(N) v(\{i\})}{v(\{i\})+v(\{j\})}$.

Next, adding continuity, which states that if two games are almost the same then their payoffs are almost the same, we can extend this result from rational numbers to real numbers.

- Continuity. For games $(N, v),(N, w) \in \mathcal{G}_{0}^{2}, \lim _{(N, w) \rightarrow(N, v)} \psi(N, w)=$ $\psi(N, v)$.

Theorem 7. A value $\psi$ on $\mathcal{G}_{0}^{2}$ satisfies grand worth additivity, the inessential game property, and continuity if and only if $\psi=P D$.

Proof. It is clear that $P D$ satisfies the three axioms. To show uniqueness, suppose that $\psi$ is a value on $\mathcal{G}_{0}^{2}$ satisfying the three axioms. From Proposition 3, we already know $\psi(N, v)=P D(N, v)$ for all $(N, v) \in \mathcal{G}_{0 \mathbb{Q}}^{2}$. Now, take any game $(N, v) \in \mathcal{G}_{0}^{2}$, and let $\left\{\left(N, v_{m}\right)\right\}$ be a sequence of games

respectively. 
in the class $\mathcal{G}_{0 \mathbb{Q}}^{2}$ such that $\lim _{m \rightarrow \infty}\left(N, v_{m}\right)=(N, v)$. Using continuity we have $\psi(N, v)=\lim _{\left(N, v_{m}\right) \rightarrow(N, v)} \psi\left(N, v_{m}\right)=\lim _{\left(N, v_{m}\right) \rightarrow(N, v)} P D\left(N, v_{m}\right)=P D(N, v)$, where the last equality holds since $P D(N, v)$ is a continuous function with respect to $(N, v) \in \mathcal{G}_{0}^{2}$.

By Theorems 6 and 7 , we immediately obtain the following corollary.

Corollary 3. The PD value is the unique value on $\hat{\mathcal{G}}_{0}$ that satisfies grand worth additivity, the inessential game property, continuity, and projection consistency.

Remark 5. Each of the following two axioms can replace grand worth additivity in Theorem 7 and characterizes the PD value together with the inessential player property on $\mathcal{G}_{0}^{2}$.

- Grand worth proportionality. For $N=\{i, j\}$, consider games $(N, v),(N, w) \in \mathcal{G}_{0}^{2}$ and take $\alpha \in \mathbb{R}$ such that $v(\{i\})=w(\{i\}), v(\{j\})=$ $w(\{j\})$ and $w(N)=\alpha v(N)$. Then $\psi(N, w)=\alpha \psi(N, v)$.

- Restricted covariance. For $N=\{i, j\}$, consider games $(N, v),(N, w) \in$ $\mathcal{G}_{0}^{2}$ and take $\alpha \in \mathbb{R}$ such that $v(\{i\})=w(\{i\}), v(\{j\})=w(\{j\})$ and $w(N)=v(N)+\alpha[v(\{i\})+v(\{j\})]$. Then $\psi_{i}(N, w)=\psi_{i}(N, v)+\alpha v(\{i\})$ and $\psi_{j}(N, w)=\psi_{j}(N, v)+\alpha v(\{j\})$.

Not surprisingly, Remark 5 and Theorem 6 imply that the PD value on $\hat{\mathcal{G}}_{0}$ is characterized by the inessential game property, projection consistency, and either grand worth proportionality or restricted covariance.

Remark 6. Ortmann (2000) introduced his proportional value that can be characterized by proportional standardness and consistency due to Hart and Mas-Colell (1989). As a consequence of Theorem 7, several characterizations of this proportional value can be obtained by replacing proportional standardness by any of its axiomatizations provided in this section. Notice that we cannot use the axiomatization as given by Proposition 1, since balanced treatment may not be satisfied by this proportional value for games with more than two players. 


\section{Conclusion}

In this paper, we provided several characterizations of the proportional division value for TU-games using explicit axioms that characterize different fairness criteria, such as equal treatment of equals, monotonicity, and consistency. It is worth noticing that balanced treatment, one of our main axioms, in some sense reflects not only equal treatment of equals but also unequal treatment of unequals. This axiom captures this feature of the proportional division value. Our axiomatic characterizations, except that for two-player games, are similar to the characterizations of the equal division value due to van den Brink (2007) and van den Brink and Funaki (2009), and the characterizations of the equal surplus division value due to Casajus and Huettner (2014). That is, most of them are obtained by weakening one axiom while strengthening the other axiom. This implies that the proportional division value is axiomatically closely related to these two equal surplus sharing values: the equal division value and the equal surplus division value.

In the future, we will study other characterizations of the proportional division value relying on some existing characterizations of the equal division value as well as the equal surplus division value. Parallel to equal surplus sharing values, we recall that the combination of the proportional division value and the equal division value for joint venture games is characterized by Moulin (1987). In this way, we mention future research on characterizations of the combination of the proportional division value and the equal division value or the equal surplus division for more general TU-games.

\section{Appendix}

This 'appendix' contains the lengthy proof of Theorem 11 (Appendix A) and the logical independence of the axioms used in characterization results (Appendix B). 


\section{Appendix A: Proof of Theorem 1}

Existence: We have to show that any value defined by (2) satisfies efficiency, weak linearity, and balanced treatment.

Summing (2) over all $i \in N$ yields

$$
\begin{aligned}
& \sum_{i \in N} \psi_{i}(N, v) \\
= & v(N)+\sum_{i \in N} \sum_{\substack{S: i \in S \neq N \\
|S| \geq 2}} \frac{v(\{i\}) \cdot \lambda_{S}}{\sum_{j \in S} v(\{j\})} v(S)-\sum_{i \in N} \sum_{\substack{S: i \notin S \\
|S| \geq 2}} \frac{v(\{i\}) \cdot \lambda_{S}}{\sum_{j \in N \backslash S} v(\{j\})} v(S) \\
= & v(N)+\sum_{\substack{S \subset N \\
|S| \geq 2}} \lambda_{S} v(S)-\sum_{\substack{S \subset N \\
|S| \geq 2}} \lambda_{S} v(S) \\
= & v(N),
\end{aligned}
$$

showing that (2) satisfies efficiency.

Consider two games $(N, v),(N, w) \in \mathcal{G}_{0}^{N}$ and $c \in \mathbb{R}_{+}$such that $w(\{i\})=$ $c v(\{i\})$ for all $i \in N$. For any $a \in \mathbb{R}$, if $(N, a v+w) \in \mathcal{G}_{0}^{N}$, we have

$$
\begin{aligned}
& \psi_{i}(N, a v+w) \\
= & \frac{(a v(\{i\})+w(\{i\}))(a v(N)+w(N))}{\sum_{j \in N}(a v(\{j\})+w(\{j\}))}+\sum_{\substack{S: i \in S \neq N \\
|S| \geq 2}} \frac{(a v(\{i\})+w(\{i\})) \cdot \lambda_{S}}{\sum_{j \in S}(a v(\{j\})+w(\{j\}))}(a v(S)+w(S)) \\
& -\sum_{\substack{S: i q S \\
|S| \geq 2}} \frac{(a v(\{i\})+w(\{i\})) \cdot \lambda_{S}}{\sum_{j \in N \backslash S}(a v(\{j\})+w(\{j\}))}(a v(S)+w(S)) \\
= & \frac{v(\{i\})(a v(N)+w(N))}{\left.\sum_{j \in N} v(\{j\})\right)}+\sum_{\substack{S: i \in S \neq N \\
|S| \geq 2}} \frac{v(\{i\}) \cdot \lambda_{S}}{\sum_{j \in S} v(\{j\})}(a v(S)+w(S)) \\
& -\sum_{\substack{S: i q S \\
|S| \geq 2}} \frac{v(\{i\}) \cdot \lambda_{S}}{\sum_{j \in N \backslash S} v(\{j\})}(a v(S)+w(S)) \\
= & \frac{v(\{i\}) a v(N)}{\sum_{j \in N} v(\{j\})}+\sum_{S: i \in S \neq N} \frac{v(\{i\}) \cdot \lambda_{S}}{\sum_{j \in S} v(\{j\})} a v(S)-\sum_{\substack{S: i \notin S \\
|S| \geq 2}} \frac{v(\{i\}) \cdot \lambda_{S}}{\sum_{j \in N \backslash S} v(\{j\})} a v(S) \\
& +\frac{c v(\{i\}) w(N)}{c \sum_{j \in N} v(\{j\})}+\sum_{\substack{S: i \in S \neq N \\
|S| \geq 2}} \frac{c v(\{i\}) \cdot \lambda_{S}}{c \sum_{j \in S} v(\{j\})} w(S)-\sum_{\substack{S: i q S \\
|S| \geq 2}} \frac{c v(\{i\}) \cdot \lambda_{S}}{c \sum_{j \in N \backslash S} v(\{j\})} w(S) \\
= & a \psi_{i}(N, v)+\psi_{i}(N, w),
\end{aligned}
$$


showing that (2) satisfies weak linearity.

Let $i, k \in N$ be two players such that $v(S \cup\{i\})=v(S \cup\{k\})$ for all $S \subseteq N \backslash\{i, k\}, S \neq \emptyset$. We have the following:

$$
\begin{aligned}
& \frac{\psi_{i}(N, v)}{v(\{i\})}-\frac{\psi_{k}(N, v)}{v(\{k\})} \\
& =\sum_{\substack{S: i \in S, k \notin S \\
|S| \geq 2}} \frac{\lambda_{S}}{\sum_{j \in S} v(\{j\})} v(S)-\sum_{\substack{S: i \notin S, k \in S \\
|S| \geq 2}} \frac{\lambda_{S}}{\sum_{j \in N \backslash S} v(\{j\})} v(S) \\
& -\left[\sum_{\substack{S: k \in S, i \notin S \\
|S| \geq 2}} \frac{\lambda_{S}}{\sum_{j \in S} v(\{j\})} v(S)-\sum_{\substack{S: k \notin S, i \in S \\
|S| \geq 2}} \frac{\lambda_{S}}{\sum_{j \in N \backslash S} v(\{j\})} v(S)\right] \\
& =\sum_{\substack{S: i, k \notin S \\
|S| \geq 1}} \frac{\lambda_{S \cup\{i\}}}{\sum_{j \in S} v(\{j\})+v(\{i\})} v(S \cup\{i\})-\sum_{\substack{S: i, k \notin S \\
|S| \geq 1}} \frac{\lambda_{S \cup\{k\}}}{\sum_{j \in N \backslash S} v(\{j\})-v(\{k\})} v(S \cup\{k\}) \\
& -\left[\sum_{\substack{S: i, k \notin S \\
|S| \geq 1}} \frac{\lambda_{S \cup\{k\}}}{\sum_{j \in S} v(\{j\})+v(\{k\})} v(S \cup\{k\})-\sum_{\substack{S: i, k \notin S \\
|S| \geq 1}} \frac{\lambda_{S \cup\{i\})}}{\sum_{j \in N \backslash S} v(\{j\})-v(\{i\})} v(S \cup\{i\}]\right. \\
& =\sum_{\substack{S: i, k \notin S \\
|S| \geq 1}} \frac{\lambda_{S \cup\{i\}}}{\sum_{j \in S} v(\{j\})+v(\{i\})} v(S \cup\{i\})+\sum_{\substack{S: i, k \notin S \\
|S| \geq 1}} \frac{\lambda_{S \cup\{i\}}}{\sum_{j \in N \backslash S} v(\{j\})-v(\{i\})} v(S \cup\{i\}) \\
& -\left[\sum_{\substack{S: i, k \notin S \\
|S| \geq 1}} \frac{\lambda_{S \cup\{k\}}}{\sum_{j \in N \backslash S} v(\{j\})-v(\{k\})} v(S \cup\{k\})+\sum_{\substack{S: i, k \notin S \\
|S| \geq 1}} \frac{\lambda_{S \cup\{k\}}}{\sum_{j \in S} v(\{j\})+v(\{k\})} v(S \cup\{k\})\right] \\
& =\sum_{\substack{S: i, k \notin S \\
|S| \geq 1}} \frac{\sum_{j \in N} v(\{j\}) \lambda_{S \cup\{i\}} v(S \cup\{i\})}{\left[\sum_{j \in S} v(\{j\})+v(\{i\})\right]\left[\sum_{j \in N \backslash S} v(\{j\})-v(\{i\})\right]}-\sum_{\substack{S: i, k \notin S \\
|S| \geq 1}} \frac{\sum_{j \in N} v(\{j\}) \lambda_{S \cup\{k\}} v(S \cup\{k\})}{\left[\sum_{j \in S} v(\{j\})+v(\{k\})\right]\left[\sum_{j \in N \backslash S} v(\{j\})-v(\{k\})\right]} \\
& =\sum_{\substack{S: i, k \notin S \\
|S| \geq 1}} \frac{\sum_{j \in N} v(\{j\}) \lambda_{S \cup\{k\}} v(S \cup\{i\})}{\left[\sum_{j \in S} v(\{j\})+v(\{k\})\right]\left[\sum_{j \in N \backslash S} v(\{j\})-v(\{k\})\right]}-\sum_{\substack{S: i, k \notin S \\
|S| \geq 1}} \frac{\sum_{j \in N} v(\{j\}) \lambda_{S \cup\{k\}} v(S \cup\{k\})}{\left[\sum_{j \in S} v(\{j\})+v(\{k\})\right]\left[\sum_{j \in N \backslash S} v(\{j\})-v(\{k\})\right]} \\
& =0 \text {, }
\end{aligned}
$$

where the fifth equality follows by (3). This implies that (2) satisfies balanced treatment.

Uniqueness: Let $\psi$ be a value satisfying efficiency, weak linearity, and balanced treatment. For $|N|=1$ and $|N|=2$, uniqueness follows from efficiency and Proposition 1 respectively. Now let $(N, v) \in \mathcal{G}_{0}^{N}$ be an arbitrary game with $|N| \geq 3$. In order to use the property of weak linearity, we decompose $(N, v)$ into the unique combination of the following two kinds of 
games $\left(N, w^{S}\right)$ and $(N, w) \sqrt{12}$, For any coalition $S \subseteq N$ with $|S| \geq 2$, the game $\left(N, w^{S}\right)$ is defined as follows:

$$
w^{S}(T)= \begin{cases}v(\{i\}), & \text { if } T=\{i\} \text { for all } i \in N \\ 1, & \text { if } T=S \\ 0, & \text { otherwise. }\end{cases}
$$

The game $(N, w)$ is defined as follows:

$$
w(T)= \begin{cases}v(\{i\}), & \text { if } T=\{i\} \text { for all } i \in N \\ 0, & \text { otherwise }\end{cases}
$$

One can easily check that $(N, v)$ can be written as $v=I(v) w+\sum_{S \subseteq N,|S| \geq 2} v(S) w^{S}$, where $I(v)=1-\sum_{S \subseteq N,|S| \geq 2} v(S)$. By using weak linearity ${ }^{13}$ of $\psi$, we have

$$
\psi_{i}(N, v)=I(v) \psi_{i}(N, w)+\sum_{S \subseteq N,|S| \geq 2} v(S) \psi_{i}\left(N, w^{S}\right), \quad \text { for all } i \in N
$$

Now, by balanced treatment, for each $S \subset N$ with $|S| \geq 2$, since all players in $S$ are weak symmetric in $\left(N, w^{S}\right)$, and the same for all players in $N \backslash S$, there must exist some $\lambda_{S}$ and $\mu_{S}$ such that

$$
\psi_{i}\left(N, w^{S}\right)= \begin{cases}\frac{v(\{i\})}{\sum_{j \in S} v(\{j\})} \lambda_{S}, & \text { if } i \in S, \\ \frac{v(\{i\})}{\sum_{j \in N \backslash S} v(\{j\})} \mu_{S}, & \text { if } i \notin S .\end{cases}
$$

\footnotetext{
${ }^{12}$ For any $(N, v) \in \mathcal{G}_{0}^{N}$, the collection of games $\left\{(N, w),\left(N, w^{S}\right)_{S \subseteq N,|S| \geq 2}\right\}$ is a basis of the class of games $\mathcal{G}_{v}^{N}=\left\{\left(N, v^{\prime}\right) \in \mathcal{G}_{0}^{N} \mid \exists c \in \mathbb{R}\right.$ such that $v^{\prime}(\{i\})=c v(\{i\})$ for all $i \in$ $N\} \cup\left\{(N, v) \in \mathcal{G}^{N} \mid v(\{i\})=0\right.$ for all $\left.i \in N\right\}$. The dimension of $\mathcal{G}_{v}^{N}$ is $2^{n}-n$. Another interesting basis can be found in the proof of Proposition 5 in Béal et al. (2018) or in van den Brink et al. (2018).

${ }^{13}$ To ensure that we stay in the class $\mathcal{G}_{0}^{N}$, we should consider the games in which their coefficients are nonzero in a suitable ordering, just like the technical approach as given by Lemma 5 in Béal et al. (2018).
} 
By efficiency, it must be $\sum_{i \in S} \frac{v(\{i\})}{\sum_{j \in S} v(\{j\})} \lambda_{S}+\sum_{i \in N \backslash S} \frac{v(\{i\})}{\sum_{j \in N \backslash S} v(\{j\})} \mu_{S}=0$, which shows $\lambda_{S}=-\mu_{S}$. Similarly, $\psi_{i}\left(N, w^{N}\right)=\frac{v(\{i\})}{\sum_{j \in N} v(\{j\})}$ for all $i \in N$. Meanwhile, we have $\psi(N, w)=0$. Putting all together we have the expression of $\psi$ as given by (2).

Accordingly, let us see that $\lambda_{S}$ only depends on the size of $S(S \neq N)$ and the worths of all singleton coalitions $\{i\}, i \in N$. Let $S \subset N, S \neq \emptyset$ with $i, j \notin S$, and consider the game $\left(N, w^{S \cup\{i\}}+w^{S \cup\{j\}}\right)$. In this game, since $i$ and $j$ are weak symmetric, it must be that $\frac{1}{v(\{i\})} \psi_{i}\left(N, w^{S \cup\{i\}}+w^{S \cup\{j\}}\right)=$ $\frac{1}{v(\{j\})} \psi_{j}\left(N, w^{S \cup\{i\}}+w^{S \cup\{j\}}\right)$. With 14 and weak linearity, we have that

$$
\begin{aligned}
& \frac{1}{\sum_{k \in S \cup\{i\}} v(\{k\})} \lambda_{S \cup\{i\}}-\frac{1}{\sum_{k \in N \backslash(S \cup\{j\})} v(\{k\})} \lambda_{S \cup\{j\}} \\
= & \frac{1}{\sum_{k \in S \cup\{j\}} v(\{k\})} \lambda_{S \cup\{j\}}-\frac{1}{\sum_{k \in N \backslash(S \cup\{i\})} v(\{k\})} \lambda_{S \cup\{i\}},
\end{aligned}
$$

from which it immediately follows that

$\frac{\lambda_{S \cup\{i\}}}{\sum_{k \in S \cup\{i\}} v(\{k\}) \sum_{k \in N \backslash(S \cup\{i\})} v(\{k\})}=\frac{\lambda_{S \cup\{j\}}}{\sum_{k \in S \cup\{j\}} v(\{k\}) \sum_{k \in N \backslash(S \cup\{j\})} v(\{k\})}$.

Therefore, whenever $S$ and $T$ are of the same size, replacing player by player, we can form a sequence with at most $s+1$ coalitions, such that the first one is $S$, and any of them is the result of replacing a player of $S$ by a player of $N \backslash S$. In this way, we conclude the relationship between $\lambda_{S}$ and $\lambda_{T}$ given by (3).

\section{Appendix B: Logical independence of the axioms}

Logical independence of the axioms in Theorem 3 can be shown by the following alternative values:

(i) The value $\psi$ on $\mathcal{G}_{0}^{N}$ defined for each $(N, v) \in \mathcal{G}_{0}^{N}$ and each $i \in N$, by

$$
\psi_{i}(N, v)=v(\{i\})
$$

satisfies all axioms, but not efficiency. 
(ii) Let $D_{v}$ be the set of all dummy players and all dummifying players in $(N, v)$. The value $\psi$ on $\mathcal{G}_{0}^{N}$ defined for each $(N, v) \in \mathcal{G}_{0}^{N}$ and each $i \in N$, by

$$
\psi_{i}(N, v)= \begin{cases}v(\{i\}), & \text { if } i \in D_{v} \\ \frac{v(\{i\})}{\sum_{j \in N \backslash D_{v}} v(\{j\})}\left[v(N)-\sum_{j \in D_{v}} v(\{j\})\right], & \text { otherwise. }\end{cases}
$$

satisfies all axioms, but not weak linearity.

(iii) The equal surplus division value ESD (Driessen and Funaki 1991) on $\mathcal{G}_{0}^{N}$ defined for each $(N, v) \in \mathcal{G}_{0}^{N}$ and each $i \in N$, by

$$
E S D_{i}(N, v)=v(\{i\})+\frac{1}{n}\left[v(N)-\sum_{j \in N} v(\{j\})\right]
$$

satisfies all axioms, but not balanced treatment.

(iv) The value $\psi$ on $\mathcal{G}_{0}^{N}$ as given by (2) with some $\lambda_{S} \neq 0$, satisfies all axioms, but not the dummifying player property.

Logical independence of the axioms in Theorem 4 can be shown by the following alternative values:

(i) The value $\psi$ on $\mathcal{G}_{0}^{N}$ defined for each $(N, v) \in \mathcal{G}_{0}^{N}$ and each $i \in N$, by $\psi_{i}(N, v)=v(\{i\})$ satisfies all axioms, but not efficiency.

(ii) The ESD value satisfies all axioms, but not balanced treatment.

(iii) The value $\psi$ on $\mathcal{G}_{0}^{N}$ defined for each $(N, v) \in \mathcal{G}_{0}^{N}$ and each $i \in N$, by

$$
\psi_{i}(N, v)=\frac{1}{2^{n-1}} \sum_{S: i \in S} \frac{v(\{i\})}{\sum_{j \in S} v(\{j\})} v(N)
$$

satisfies all axioms, but neither weak coalitional surplus equivalence nor weak coalitional surplus monotonicity. 
Logical independence of the axioms in Theorem 5 can be shown by the following alternative values:

(i) The value $\psi_{i}(N, v)=v(\{i\}), i \in N$, satisfies all axioms, but not efficiency.

(ii) The ED value satisfies all axioms, but not weak balanced treatment.

(iii) The value $\psi$ on $\mathcal{G}_{0}^{N}$ defined for each $(N, v) \in \mathcal{G}_{0}^{N}$ and each $i \in N$, by

$$
\psi_{i}(N, v)= \begin{cases}\frac{v(\{i\})}{\sum_{j \in N} v(\{j\})} v(N), & \text { if } v(N \backslash\{j\})=0 \text { for some } j \in N, \\ \frac{v(\{i\})}{v(N \backslash\{i\})} \frac{1}{\sum_{j \in N} \frac{v(\{j\}\})}{v(N \backslash\{j\})}} v(N), & \text { otherwise. }\end{cases}
$$

satisfies all axioms, but not weak coalitional monotonicity.

Logical independence of the axioms in Theorem 7 can be shown by the following alternative values:

(i) The value $\psi$ given by $\psi_{i}(N, v)=v(\{i\})$ for all $i \in N$ and $(N, v) \in \mathcal{G}_{0}^{2}$ satisfies the inessential game property and continuity, but not grand worth additivity.

(ii) The ED value on $\mathcal{G}_{0}^{2}$ satisfies grand worth additivity and continuity, but not the inessential game property.

(iii) The value $\psi$ given by

$$
\psi_{i}(N, v)= \begin{cases}P D_{i}(N, v), & \text { if } v(N) \in \mathbb{Q}, \\ E S D_{i}(N, v), & \text { if } v(N) \notin \mathbb{Q} .\end{cases}
$$

for all $i \in N$ and $(N, v) \in \mathcal{G}_{0}^{2}$ satisfies grand worth additivity and the inessential game property, but not continuity. 


\section{Acknowledgement}

Youngsub Chun is supported by the National Research Foundation of Korea Grant funded by the Korean Government (NRF-2016S1A3A2924944). Zhengxing Zou thanks the financial support of the National Natural Science Foundation of China (Grant Nos. 71771025, 71801016) and the China Scholarship Council (Grant No. 201806030046).

\section{References}

Banker, R., 1981. Equity considerations in traditional full cost ailocation practices: an axiomatic perspective, in: Moriarty, S. (Ed.), Joint Cost Allocations. University of Oklahoma, pp. 110-130.

Béal, S., Casajus, A., Huettner, F., Rémila, E., Solal, P., 2016. Characterizations of weighted and equal division values. Theory and Decision 80, 649-667.

Béal, S., Ferrières, S., Rémila, E., Solal, P., 2018. The proportional Shapley value and applications. Games and Economic Behavior 108, 93-112.

Besner, M., 2019. Axiomatizations of the proportional Shapley value. Theory and Decision 86, 161-183.

Calleja, P., Llerena, F., 2017. Rationality, aggregate monotonicity and consistency in cooperative games: some (im) possibility results. Social Choice and Welfare 48, 197-220.

Calleja, P., Llerena, F., 2019. Path monotonicity, consistency and axiomatizations of some weighted solutions. International Journal of Game Theory 48, 287-310.

Carreras, F., Owen, G., 2013. Pure bargaining problems and the Shapley rule, in: Holler, M., Nurmi, H. (Eds.), Power, Voting, and Voting Power: 30 Years After. Springer, pp. 681-702. 
Casajus, A., Huettner, F., 2014. Null, nullifying, or dummifying players: The difference between the Shapley value, the equal division value, and the equal surplus division value. Economics Letters 122, 167-169.

Chun, Y., 1988. The proportional solution for rights problems. Mathematical Social Sciences 15, 231-246.

Driessen, T., Funaki, Y., 1991. Coincidence of and collinearity between game theoretic solutions. OR-Spektrum 13, 15-30.

Hart, S., Mas-Colell, A., 1989. Potential, value, and consistency. Econometrica , 589-614.

Kamijo, Y., Kongo, T., 2015. Properties based on relative contributions for cooperative games with transferable utilities. Theory and Decision 78 , $77-87$.

Khmelnitskaya, A.B., Driessen, T.S., 2003. Semiproportional values for TU games. Mathematical Methods of Operations Research 57, 495-511.

Moriarity, S., 1975. Another approach to allocating joint costs. The Accounting Review 50, 791-795.

Moulin, H., 1987. Equal or proportional division of a surplus, and other methods. International Journal of Game Theory 16, 161-186.

Ortmann, K.M., 2000. The proportional value for positive cooperative games. Mathematical Methods of Operations Research 51, 235-248.

Ruiz, L.M., Valenciano, F., Zarzuelo, J.M., 1998. The family of least square values for transferable utility games. Games and Economic Behavior 24, $109-130$.

Shapley, L.S., 1953. A value for n-person games, in: Kuhn, H., Tucked, A. (Eds.), Contributions to the Theory of Games. Princeton University Press. volume 28 of 2 , pp. 307-317. 
Shubik, M., 1962. Incentives, decentralized control, the assignment of joint costs and internal pricing. Management Science 8, 325-343.

van den Brink, R., 2007. Null or nullifying players: the difference between the Shapley value and equal division solutions. Journal of Economic Theory 136, 767-775.

van den Brink, R., Chun, Y., Funaki, Y., Park, B., 2016. Consistency, population solidarity, and egalitarian solutions for TU-games. Theory and Decision 81, 427-447.

van den Brink, R., Funaki, Y., 2009. Axiomatizations of a class of equal surplus sharing solutions for TU-games. Theory and Decision 67, 303-340.

van den Brink, R., Levínskỳ, R., Zelenỳ, M., 2015. On proper Shapley values for monotone TU-games. International Journal of Game Theory 44, 449471.

van den Brink, R., Levínskỳ, R., Zelenỳ, M., 2018. The Shapley value, proper Shapley value, and sharing rules for cooperative ventures. Tinbergen Institute Discussion Paper 2018-089/II.

Vorob'ev, N., Liapunov, A., 1998. The proper Shapley value. Game theory and applications 4, 155-159.

Yokote, K., Funaki, Y., 2017. Monotonicity implies linearity: characterizations of convex combinations of solutions to cooperative games. Social Choice and Welfare 49, 171-203.

Young, H.P., 1985. Monotonic solutions of cooperative games. International Journal of Game Theory 14, 65-72. 